Originalien

Anaesthesist 2021 · 70:383-391

https://doi.org/10.1007/s00101-020-00889-1

Eingegangen: 11. Juli 2020

Überarbeitet: 23. September 2020

Angenommen: 27. Oktober 2020

Online publiziert: 26 . November 2020

(c) Der/die Autor(en) 2020

Marc Felzen' · Hanna Schröder' · Stefan K. Beckers' Nadine Rott ${ }^{2} \cdot$ Ruth Koch-Schultze ${ }^{2} \cdot$ Sabine Wingen ${ }^{2} \cdot$ Andreas Meißner $^{3}$. Iris Santowski ${ }^{4}$. Olaf Picker ${ }^{4} \cdot$ Niels Rahe-Meyer ${ }^{5} \cdot$ Rico Dumcke $^{6} \cdot$ Claas Wegner $^{6}$. Hugo van Aken ${ }^{7}$. Antje Gottschalk ${ }^{7} \cdot$ Oliver Weber $^{8} \cdot$ Rolf Rossaint $^{1}$

' Klinik für Anästhesiologie, Medizinische Fakultät, RWTH Aachen University, Uniklinik RWTH Aachen, Aachen, Deutschland

${ }^{2}$ Klinik für Anästhesiologie und Operative Intensivmedizin, Universitätsklinikum Köln, Köln, Deutschland

${ }^{3}$ Klinik für Anästhesiologie, Klinikum Stadt Soest, Soest, Deutschland

${ }^{4}$ Klinik für Anästhesiologie, Universitätsklinikum Düsseldorf, Düsseldorf, Deutschland

${ }^{5}$ Klinik für Anästhesiologie und operative Intensivmedizin, Franziskus Hospital, Bielefeld, Deutschland

${ }^{6}$ Fakultät für Biologie, Biologiedidaktik, Universität Bielefeld, Bielefeld, Deutschland

${ }^{7}$ Klinik für Anästhesiologie, operative Intensivmedizin und Schmerztherapie, Universitätsklinikum Münster, Münster, Deutschland

${ }^{8}$ Zentrum für Anästhesiologie, Intensivmedizin und Schmerztherapie, Klinikum Vest, Recklinghausen, Deutschland

\title{
Evaluation des Projekts zur Einführung von Laienreanimation an Schulen in Nordrhein-Westfalen
}

\section{Hintergrund}

Wiederbelebungsunterricht ist an Schulen in Nordrhein-Westfalen (NRW) und auch deutschlandweit bisher nicht etabliert; dieser beschränkt sich auf einzelne Projekte, welche allerdings allesamt erfolgreich sind $[8,10,14]$. Dabei hat der Schulausschuss der Kultusministerkonferenz bereits im Juni 2014 die Einführung von Modulen zum Thema Wiederbelebung befürwortet und den Ländern empfohlen, Lehrkräfte entsprechend ausbilden zu lassen. Darüber hinaus soll laut Koalitionsvertrag NRW zwischen der Christlich Demokratischen Union Deutschlands (CDU) und der Freien Demokratischen Partei (FDP) 2017-2022 durch die Unterrichtung an allen Schulen in Nordrhein-Westfalen die Bereitschaft zu Erster Hilfe und Wiederbelebung von Anfang an gefördert werden. Hintergrund ist eine niedrige Laienreanimationsrate in Deutschland, welche $z u$ geringen Überlebenschancen eines Herzstillstands führt. Ursächlich dafür ist das Absterben von Hirnzellen bereits $5 \mathrm{~min}$ nach dem Herzstillstand ohne Wiederbelebungsmaßnahmen, also meistens noch vor Eintreffen des Rettungsdienstes. Durch unterschiedliche Bestrebungen ist die Laienreanimationsrate in den letzten Jahren bereits von $17 \%$ auf 39\% [35] angestiegen, Deutschland liegt jedoch im europäischen Vergleich immer noch deutlich unterhalb des Durchschnitts von $58 \%$ [16]. So konnte die Laienreanimationsrate in Dänemark durch die landesweite Einführung von Pflichtunterricht zum Thema Wiederbelebung von $20 \%$ im Jahre 2001 auf mehr als $50 \%$ im Jahre 2012 gesteigert werden [24]. Während Wiederbelebungsunterricht in Belgien, Dänemark, Frankreich, Italien und Portugal im Rahmen der globalen, von der Weltgesundheitsorganisation unterstützten, Initiative „Kids save lives" $[9,11,12]$ gesetzlich verankert ist, wird er in den meisten Ländern bisher lediglich empfohlen. In Deutschland existieren bisher nur für die Bundesländer Baden-Württemberg über die im September 2015 gestartete Initiative „Löwen retten Leben“, Bayern über 


\begin{tabular}{|c|c|c|c|c|c|c|}
\hline Regierungsbezirk & Arnsberg & Detmold & Köln & & Düsseldorf & Münster \\
\hline Koordinierende Klinik & $\begin{array}{l}\text { Klinikum Stadt } \\
\text { Soest }\end{array}$ & $\begin{array}{l}\text { Franziskus Hospi- } \\
\text { tal Bielefeld }\end{array}$ & Uniklinik Aachen & Uniklinik Köln & $\begin{array}{l}\text { Uniklinik } \\
\text { Düsseldorf }\end{array}$ & $\begin{array}{l}\text { Uniklinik } \\
\text { Münster }\end{array}$ \\
\hline Gesamtdauer [min] & 90 & 90 & 45 & 90 & 90 & 90 \\
\hline Dauer, Theorie [min] & 45 & 45 & 20 & 45 & 45 & 45 \\
\hline Dauer, Praxis [min] & 45 & 45 & 25 & 45 & 45 & 45 \\
\hline Dozenten & Lehrer & Lehrer & Notärzte & Lehrer & Lehrer & Lehrer \\
\hline Anzahl, Schüler/ Durchgang & 1 Klasse & 1 Jahrgang & 1 Jahrgang & 1 Klasse & 1 Klasse & 1 Klasse \\
\hline Training & $\begin{array}{l}\text { Herzdruck- } \\
\text { massage }\end{array}$ & $\begin{array}{l}\text { Herzdruck- } \\
\text { massage } \\
\text { (ggf. + Beat- } \\
\text { mung + AED) }\end{array}$ & $\begin{array}{l}\text { Herzdruck- } \\
\text { massage }\end{array}$ & $\begin{array}{l}\text { Herzdruck- } \\
\text { massage }\end{array}$ & $\begin{array}{l}\text { Herzdruck- } \\
\text { massage }\end{array}$ & $\begin{array}{l}\text { Herzdruck- } \\
\text { massage }\end{array}$ \\
\hline Wiederholung & 2-jährlich & Jährlich & Jährlich & Jährlich & Jährlich & Jährlich \\
\hline
\end{tabular}

\begin{tabular}{|c|c|c|c|c|c|c|}
\hline \multirow{2}{*}{ Regierungsbezirk } & \multirow[t]{2}{*}{ Arnsberg } & \multirow{2}{*}{ Detmold } & \multicolumn{2}{|l|}{ Köln } & \multirow{2}{*}{$\begin{array}{l}\text { Düssel- } \\
\text { dorf }\end{array}$} & \multirow[t]{2}{*}{ Münster } \\
\hline & & & Aachen & Köln & & \\
\hline \multicolumn{7}{|l|}{ Zahlen } \\
\hline Schulen & 65 & 67 & 23 & 26 & 26 & 42 \\
\hline Jahrgang & $7+8$ & $7-12$ & $7-12$ & $7-12$ & $7-12$ & $7-12$ \\
\hline Schüler & 9000 & 5029 & 10634 & 8249 & 1952 & $>6000$ \\
\hline Lehrer & 437 & 194 & 11 & 223 & 39 & 110 \\
\hline Studenten & 0 & 72 & 17 & 0 & 0 & 0 \\
\hline \multicolumn{7}{|l|}{ Dozenten } \\
\hline Lehrer & $v$ & $\checkmark$ & $\square$ & $\checkmark$ & $\checkmark$ & $\checkmark$ \\
\hline Studenten & $\boldsymbol{\nu}^{\mathrm{a}}$ & $\boldsymbol{V}^{\mathrm{b}}$ & $\square$ & $\square$ & $\square$ & $\square$ \\
\hline Ärzte & $\square$ & $\square$ & $\checkmark$ & $\square$ & $\square$ & $\square$ \\
\hline Hilfsorganisation & $\square$ & $\square$ & $\square$ & $\square$ & $\square$ & $\square$ \\
\hline
\end{tabular}

eine Bekanntmachung des Bayerischen Staatsministeriums für Unterricht und Kultus und Mecklenburg-Vorpommern über das Projekt „Retten macht Schule“ bundeslandweite Konzepte [1, 3, 22, 31], welche die Ausbildung von Lehrern für das Training von Schülern vorsehen. Diesbezüglich existieren bereits Ausbildungscurricula für Lehrer $[11,29]$ und Schüler seitens des Deutschen Rates für Wiederbelebung (GRC), deren Effektivität bereits gezeigt werden konnte $[6,8$, 10, 14, 27].

Zur Erhöhung der Laienreanimationsrate in NRW hat das Ministerium für Schule und Weiterbildung NRW per Runderlass vom 20.03.2017 [2] zum ersten August 2017 das Projekt „Laienreanimation an Schulen in NRW“ initiiert, welches von der Deutschen Gesellschaft für Anästhesiologie, dem
Berufsverband Deutscher Anästhesisten sowie dem GRC unterstützt wird. Die Laufzeit des Projekts betrug 3 Jahre. Ziel war es, möglichst viele an Schule beteiligte Personen, insbesondere Schülerinnen und Schüler, aber auch Lehr- und Fachkräfte sowie Eltern mit Wiederbelebungsmaßnahmen vertraut $\mathrm{zu}$ machen sowie für die regelmäßige Durchführung dieser zu gewinnen. Ziel dieser Arbeit ist die Evaluation dieses Projekts, verbunden mit der Fragestellung, wie Wiederbelebungsunterricht in Schulen flächendeckend eingeführt werden kann.

\section{Methode}

Unter Federführung des Ministeriums für Schule und Bildung (MSB) NRW wurde eine Steuergruppe gebildet, beste- hend aus der Referatsleitung Gesundheit des MSB, Schuldezernenten der 5 NRWRegierungsbezirke, ärztlichen Partnern aus allen 5 NRW-Regierungsbezirken (• Tab. 1) sowie dem Referatsleiter Rettungswesen des Ministeriums für Arbeit, Gesundheit und Soziales (MAGS) NRW und einem Vertreter des Betriebskrankenkassen Landesverband Nordwest (BKK LV Nordwest).

Zunächst wurden alle weiterführenden Schulen in NRW schriftlich zur Teilnahme am Projekt eingeladen. Das MAGS und der BKK LV Nordwest stellten Spendengelder für die Anschaffung von Wiederbelebungspuppen zur Verfügung. Nach Durchführung einer Ausschreibung wurde das Modell „PractiMan“ (Fa. Vimetecsa, Alicante, Spanien) beschafft und in Klassensätzen an alle teilnehmenden Schulen ausgeliefert.

Die Durchführung des Projektes erfolgte mithilfe von durch die ärztlichen Partner in den einzelnen Regierungsbezirken bereits etablierten unterschiedlichen Schulungskonzepten. Diese sahen entweder eine 2- bis 4-stündige Schulung von Lehrern in Anlehnung an den modularen Lehrerausbildungskurs des GRC [13] zum Trainieren der Schüler oder aber ein direktes Training von Schülern vor. Grundlage bildete die Trias „Prüfen - Rufen - Drücken“ der Aktion „Ein Leben retten“ [4], standortabhängig erweitert um Beatmung und automatischen externen Defibrillator (•Tab. 1). Sämtliches Unterrichtsmaterial wurde den Lehrern zur Verfügung gestellt. Weder Lehrer noch Schüler erhielten im Rahmen des Projekts einen zertifizierten Erste-Hilfe- 
Anaesthesist 2021 · 70:383-391 https://doi.org/10.1007/s00101-020-00889-1

(c) Der/die Autor(en) 2020

M. Felzen · H. Schröder · S. K. Beckers · B. W. Böttiger · N. Rott · R. Koch-Schultze · S. Wingen · A. Meißner · I. Santowski · O. Picker · N. Rahe-Meyer · R. Dumcke · C. Wegner · H. van Aken · A. Gottschalk · O. Weber · R. Rossaint

\section{Evaluation des Projekts zur Einführung von Laienreanimation an Schulen in Nordrhein-Westfalen}

\section{Zusammenfassung}

Hintergrund. Wiederbelebungsunterricht ist nicht in allen Schulen Deutschlands verpflichtend, dieser beschränkt sich trotz niedriger Laienreanimationsrate aktuell auf einzelne, z.T verpflichtende Projekte in unterschiedlichen Bundesländern. Aus diesem Grunde hat das Ministerium für Schule und Bildung Nordrhein-Westfalen per Runderlass im März 2017 das Projekt "Laienreanimation an Schulen in NRW“ initiiert.

Fragestellung. Ziel dieser Arbeit ist die Evaluation dieses Projekts.

Material und Methoden. Alle weiterführenden Schulen in Nordrhein-Westfalen wurden zur Teilnahme am Projekt eingeladen. Aus jedem Regierungsbezirk nahmen ärztliche Partner teil, welche Wiederbelebungstrainings mit bereits bestehenden Konzepten zum Lehrer- oder Schülertraining durchführten. Nach einer 3-jährigen Laufzeit erfolgte die Evaluation anhand von standardisierten Fragebögen für Schuldezernenten, Lehrer und Schüler.

Ergebnisse. Insgesamt konnten durch das Projekt mehr als 40.000 Schüler aus 249 Schulen in NRW mit 6 unterschiedlichen Konzepten in Wiederbelebung trainiert werden. Fragen bezüglich Wiederbelebungsmaßnahmen konnten durch $85 \%$ der Schüler richtig beantwortet werden. Die Schüler fühlen sich insgesamt sicher in Wiederbelebungsmaßnahmen. Der Investitionsbedarf für alle Schulen liegt bei einmalig zwischen 4 und 6,5 Mio. $€$ sowie rund $340.000 €$ in jedem Haushaltsjahr. Diskussion. Eine gesetzliche Verpflichtung und Finanzierung von Wiederbelebungstrainings sind unerlässlich für eine flächendeckende Durchführung. Alle durchgeführten Konzepte sind effektiv, dementsprechend kann jede Schule ein auf ihre Bedürfnisse abgestimmtes Konzept auswählen und dieses bestenfalls gestuft anwenden. Die Schulung von Lehrern sollte gezielt auf Wiederbelebung ausgerichtet sein.

Schlüsselwörter

Wiederbelebung in Schulen · Ein Leben retten . Herzstillstand · Erste Hilfe · Ministerium für Schule und Bildung

\section{Evaluation of the project for the introduction of bystander resuscitation in schools in North Rhine- Westphalia}

\section{Abstract}

Background. Teaching of resuscitation measures is not mandatory in all schools in Germany. It is currently limited to individual, partly mandatory projects despite a low bystander resuscitation rate. For this reason, the Ministry for Schools and Education of North Rhine-Westphalia initiated the project "Bystander resuscitation at schools in NRW" in March 2017.

Objective. The aim of this work was to evaluate this project.

Material and methods. All secondary schools in North Rhine-Westphalia were invited to participate in the project. Medical partners from each administrative district took part, who carried out resuscitation training with existing concepts for teacher or student training. After a 3-year period, the evaluation was carried out using standardized questionnaires for school headmasters, teachers and students.

Results. In total, more than 40,000 pupils from 249 schools in NRW could be trained in resuscitation within the project with 6 different concepts. Of the students $85 \%$ answered the questions regarding resuscitation correctly and overall felt safe in resuscitation measures. The one-off investment requirement for all schools is roughly 4-6.5 million $€$ and around $340,000 €$ in each budget year.

Conclusion. A legal constitution and funding are necessary for a nationwide introduction of resuscitation in schools. All established concepts are effective, therefore each school can use them exactly according to their needs, optimally in a stepped form. Training for teachers should focus on resuscitation.

Keywords

Resuscitation in schools · Safe a live · Cardiac arrest · First aid · Ministry for schols and education
Kurs; Fokus der Schulungen war die Wiederbelebung.

Die Evaluation erfolgte anhand von standardisierten Fragebögen für die Schuldezernenten, Lehrer und Schüler (Anlage 1-3), wobei die Lehrer- und Schülerbefragung nur stichprobenartig in allen Regierungsbezirken erfolgte.

Signifikanztestungen erfolgten mit dem Exakten Test nach Fisher auf einem Signifikanzniveau von $5 \%$.

\section{Ergebnisse}

Insgesamt konnten zwischen August 2017 und August 2020 durch das Projekt mit 6 bestehenden Konzepten mehr als 40.000 Schüler in NRW in Wiederbelebung trainiert werden. Es nahmen 249 (9,2\%) der 2708 weiterführenden Schulen in NRW am Projekt teil. Darüber hinaus wurden 1103 Multiplikatoren (1014 Lehrer, 89 Studenten) ausgebildet.

Mithilfe von Spendengeldern konnten nach Ausschreibung 2974 Wiederbelebungspuppen Practi-Man der Fa. Vime- tecsa sowie für den Regierungsbezirk Münster 600 Mini Anne (Fa. Laerdal, Puchheim, Deutschland) beschafft und an die Schulen verteilt werden. Der Practi-Man zeigte sich robust auch nach Durchführung von mehreren Wiederbelebungstrainings. Einzig die individuelle Nachbestellung von Beatmungszubehör durch die Schulen gestaltete sich schwierig. Die Mini Anne wurde den Schülern nach dem Training geschenkt und dementsprechend nicht wiederverwendet. 


\section{Originalien}

\begin{tabular}{|c|c|c|c|c|c|c|c|c|}
\hline \multirow[t]{2}{*}{ Frage } & & \multirow[t]{2}{*}{ Arnsberg } & \multirow[t]{2}{*}{ Detmold } & \multicolumn{2}{|l|}{ Köln } & \multirow[t]{2}{*}{ Düsseldorf } & \multirow[t]{2}{*}{ Münster } & \multirow[t]{2}{*}{ Gesamt } \\
\hline & & & & Aachen & Köln & & & \\
\hline Anzahl & & $19(100 \%)$ & 19 (100\%) & $12(100 \%)$ & $3(100 \%)$ & 12 (100\%) & $8(100 \%)$ & $73(100 \%)$ \\
\hline \multirow[t]{4}{*}{ Relevanz } & Sehr hoch & $6(31,6 \%)$ & $6(31,6 \%)$ & $1(8,3 \%)$ & $2(66,7 \%)$ & $7(58,3 \%)$ & $4(50,0 \%)$ & $26(41,1 \%)$ \\
\hline & Eher hoch & $12(63,2 \%)$ & $6(31,6 \%)$ & $4(33,3 \%)$ & $0(0,0 \%)$ & $3(25,0 \%)$ & $3(37,5 \%)$ & $28(31,8 \%)$ \\
\hline & Eher gering & $1(5,3 \%)$ & $4(21,1 \%)$ & $3(25,0 \%)$ & $1(33,3 \%)$ & $1(8,3 \%)$ & $1(12,5 \%)$ & $11(17,6 \%)$ \\
\hline & Sehr gering & $0(0,0 \%)$ & $2(10,5 \%)$ & $4(33,3 \%)$ & $0(0,0 \%)$ & $3(25,0 \%)$ & $0(0,0 \%)$ & $9(11,5 \%)$ \\
\hline \multirow[t]{4}{*}{ Motivation } & Sehr hoch & 11 (57,9\%) & $5(26,3 \%)$ & $4(33,3 \%)$ & $2(66,7 \%)$ & $4(33,3 \%)$ & $4(50,0 \%)$ & $30(44,6 \%)$ \\
\hline & Eher hoch & $6(31,6 \%)$ & $8(42,1 \%)$ & $3(25,0 \%)$ & $0(0,0 \%)$ & $6(50,0 \%)$ & $3(37,5 \%)$ & $26(31,0 \%)$ \\
\hline & Eher gering & $2(10,5 \%)$ & $6(31,6 \%)$ & $5(41,7 \%)$ & $1(33,3 \%)$ & $1(8,3 \%)$ & $0(0,0 \%)$ & $15(20,9 \%)$ \\
\hline & Sehr gering & $0(0,0 \%)$ & $0(0,0 \%)$ & $0(0,0 \%)$ & $0(0,0 \%)$ & $1(8,3 \%)$ & $0(0,0 \%)$ & $1(1,4 \%)$ \\
\hline \multirow{2}{*}{$\begin{array}{l}\text { Bereits Schüler } \\
\text { trainiert }\end{array}$} & Ja & 15 (78,9\%) & $16(84,2 \%)$ & $12(100,0 \%)$ & $3(100,0 \%)$ & $8(66,7 \%)$ & $7(87,5 \%)$ & $61(86,2 \%)$ \\
\hline & Nein & $4(21,1 \%)$ & $3(15,8 \%)$ & $1(8,3 \%)$ & $0(0,0 \%)$ & $4(33,3 \%)$ & $0(0,0 \%)$ & $12(13,1 \%)$ \\
\hline \multirow{2}{*}{$\begin{array}{l}\text { Mit Lehrplan } \\
\text { verbunden }\end{array}$} & Ja & $9(47,4 \%)$ & $11(57,9 \%)$ & $5(41,7 \%)$ & $1(33,3 \%)$ & $4(33,3 \%)$ & $1(12,5 \%)$ & $31(37,7 \%)$ \\
\hline & Nein & $10(52,6 \%)$ & $8(42,1 \%)$ & $7(58,3 \%)$ & $2(66,7 \%)$ & $8(66,7 \%)$ & $6(75,0 \%)$ & $41(60,2 \%)$ \\
\hline \multirow[t]{3}{*}{ Durchführung $^{\mathrm{a}}$} & Schulstunden & $10(52,6 \%)$ & $9(47,4 \%)$ & $1(8,3 \%)$ & - & $12(100,0 \%)$ & $3(37,5 \%)$ & $35(41,0 \%)$ \\
\hline & Aktionstage & $8(42,1 \%)$ & $7(36,8 \%)$ & $3(25,0 \%)$ & - & $1(8,3 \%)$ & $5(62,5 \%)$ & $24(29,1 \%)$ \\
\hline & Projektwoche & $0(0,0 \%)$ & $5(26,3 \%)$ & $0(0,0 \%)$ & - & $2(16,7 \%)$ & $1(12,5 \%)$ & $8(9,2 \%)$ \\
\hline \multirow{2}{*}{$\begin{array}{l}\text { Ausreichend } \\
\text { vorbereitet zu } \\
\text { unterrichten }\end{array}$} & $\mathrm{Ja}$ & $13(68,4 \%)$ & $10(52,6 \%)$ & $4(33,3 \%)$ & $2(66,7 \%)$ & $9(75,0 \%)$ & $5(62,5 \%)$ & $43(59,8 \%)$ \\
\hline & Nein & $6(31,6 \%)$ & $9(47,4 \%)$ & $8(66,7 \%)$ & $1(33,3 \%)$ & $3(25,0 \%)$ & $1(12,5 \%)$ & $28(36,1 \%)$ \\
\hline \multirow{4}{*}{$\begin{array}{l}\text { Zufriedenheit } \\
\text { ärztliche Partner }\end{array}$} & Sehr zufrieden & $7(36,8 \%)$ & $9(47,4 \%)$ & $8(66,7 \%)$ & - & $7(58,3 \%)$ & $3(37,5 \%)$ & $34(41,1 \%)$ \\
\hline & Eher zufrieden & $4(21,1 \%)$ & $7(36,8 \%)$ & $0(0,0 \%)$ & - & $5(41,7 \%)$ & $2(25,0 \%)$ & $18(20,8 \%)$ \\
\hline & Eher nicht zufrieden & $0(0,0 \%)$ & $0(0,0 \%)$ & $0(0,0 \%)$ & - & $0(0,0 \%)$ & $0(0,0 \%)$ & $0(0,0 \%)$ \\
\hline & Gar nicht zufrieden & $1(5,3 \%)$ & $0(0,0 \%)$ & $0(0,0 \%)$ & - & $0(0,0 \%)$ & $1(12,5 \%)$ & $2(3,0 \%)$ \\
\hline \multirow[t]{5}{*}{ Unterrichtsmaterial $^{\mathrm{a}}$} & Arbeitsblätter & $14(73,7 \%)$ & $16(84,2 \%)$ & $4(33,3 \%)$ & $3(100,0 \%)$ & $7(58,3 \%)$ & $7(87,5 \%)$ & $51(72,8 \%)$ \\
\hline & Flyer & $9(47,4 \%)$ & 15 (78,9\%) & $0(0,0 \%)$ & $0(0,0 \%)$ & $1(8,3 \%)$ & $5(62,5 \%)$ & $30(32,9 \%)$ \\
\hline & $\begin{array}{l}\text { Informations- } \\
\text { broschüren }\end{array}$ & $9(47,4 \%)$ & $13(68,4 \%)$ & $4(33,3 \%)$ & $1(33,3 \%)$ & $4(33,3 \%)$ & $5(62,5 \%)$ & $36(46,4 \%)$ \\
\hline & Funktionsmodelle & $8(42,1 \%)$ & $13(68,4 \%)$ & $4(33,3 \%)$ & $1(33,3 \%)$ & $4(33,3 \%)$ & $5(62,5 \%)$ & $35(45,5 \%)$ \\
\hline & Sonstiges & $0(0,0 \%)$ & $0(0,0 \%)$ & $1(8,3 \%)$ & $0(0,0 \%)$ & $0(0,0 \%)$ & $0(0,0 \%)$ & $1(1,4 \%)$ \\
\hline \multirow{2}{*}{$\begin{array}{l}\text { Leitfäden } \\
\text { gewünscht }\end{array}$} & Ja & $12(63,2 \%)$ & 15 (78,9\%) & $10(83,3 \%)$ & $3(100,0 \%)$ & $8(66,7 \%)$ & 7 (87,5\%) & $55(79,9 \%)$ \\
\hline & Nein & $7(36,8 \%)$ & $3(15,8 \%)$ & $2(16,7 \%)$ & $0(0,0 \%)$ & $4(33,3 \%)$ & $0(0,0 \%)$ & $16(17,1 \%)$ \\
\hline \multirow{2}{*}{$\begin{array}{l}\text { Verbindliches } \\
\text { Konzept }\end{array}$} & Ja & $9(47,4 \%)$ & 7 (36,8\%) & $3(25,0 \%)$ & $2(66,7 \%)$ & $1(8,3 \%)$ & $1(12,5 \%)$ & $23(32,8 \%)$ \\
\hline & Nein & $10(52,6 \%)$ & $10(52,6 \%)$ & $9(75,0 \%)$ & $0(0,0 \%)$ & $11(91,7 \%)$ & $7(87,5 \%)$ & $47(59,9 \%)$ \\
\hline
\end{tabular}

\section{Lehrerbefragung}

Es liegen Ergebnisse von 71 Lehrkräften vor. Die Lehrkräfte wünschen sich eine verpflichtende Verankerung des Wiederbelebungsunterrichts im Lehrplan sowie einen Start bereits in der Grundschule, verbunden mit einem fertigen Konzept, Praxishilfen und Leitfäden. Weiterhin sollen Schulsanitäter zur Unterstützung der Wiederbelebungstrainings ausgebildet werden. Darüber hinaus sind die Lehrer der Meinung, dass Laienreanimation nicht privaten Anbietern überlassen werden kann, da die Schüler trotz eines solchen Kurses nicht handlungsfähig sind.

Aus - Tab. 3 geht hervor, dass die Lehrer die Relevanz des Themas durchschnittlich als hoch einschätzen und dementsprechend motiviert sind.

Die meisten Lehrer sind zufrieden mit den Schulungen der ärztlichen Partner und fühlen sich ausreichend vorbereitet, Schüler $\mathrm{zu}$ trainieren, und haben dies auch bereits vornehmlich in Schulstunden getan. Wiederbelebungstrainings sind jedoch größtenteils noch nicht im
Lehrplan integriert. Auch fehlt es den Lehrern an einem verbindlichen Konzept. Darüber hinaus sind Informationsmaterial und ein Leitfaden gewünscht.

\section{Schülerbefragung}

Insgesamt konnten 1657 Schülerfragebögen aller Schulformen ausgewertet werden. Aus - Tab. 4 geht hervor, dass mindestens $85 \%$ der Schüler aller Regierungsbezirke, welche den Fragebogen abgegeben haben, die Fragen bezüglich Erkennen des Kreislaufstillstands 


\begin{tabular}{|c|c|c|c|c|c|c|c|c|}
\hline \multirow[t]{2}{*}{ Frage } & & \multirow[t]{2}{*}{ Arnsberg } & \multirow[t]{2}{*}{ Detmold } & \multicolumn{2}{|l|}{ Köln } & \multirow[t]{2}{*}{ Düsseldorf } & \multirow[t]{2}{*}{ Münster } & \multirow[t]{2}{*}{ Gesamt } \\
\hline & & & & Aachen & Köln & & & \\
\hline \multicolumn{2}{|l|}{ Anzahl, Schüler } & 305 & 479 & 213 & 294 & 311 & 55 & 1657 \\
\hline \multicolumn{2}{|c|}{ Anzahl, Schulen [\% des Regierungsbezirks] } & $6(2,0 \%)$ & $6(1,3 \%)$ & $3(1,4 \%)$ & $2(0,7 \%)$ & $3(1,0 \%)$ & $1(1,8 \%)$ & $21(1,3 \%)$ \\
\hline \multicolumn{2}{|l|}{ Durchschnittsalter } & 14 & 15 & 14 & 14 & 17 & 15 & 15 \\
\hline \multicolumn{2}{|l|}{ Alter von/bis } & $12-17$ & $10-32$ & $10-20$ & $10-22$ & $10-33$ & $14-20$ & $10-33$ \\
\hline \multicolumn{9}{|c|}{ [Alle folgenden Angaben in Relation zur Anzahl der Schüler] } \\
\hline \multirow[t]{2}{*}{ Geschlecht } & Weiblich & $35,70 \%$ & $61,60 \%$ & $45,50 \%$ & $56,10 \%$ & $70,40 \%$ & $67,30 \%$ & $56,10 \%$ \\
\hline & Männlich & $29,80 \%$ & $38,40 \%$ & $54,50 \%$ & $43,50 \%$ & $27,00 \%$ & $32,70 \%$ & $37,70 \%$ \\
\hline \multirow[t]{2}{*}{ Vorher teilgenommen } & Ja & $53,80 \%$ & $72,20 \%$ & $64,80 \%$ & $86,70 \%$ & $60,50 \%$ & $89,10 \%$ & $71,20 \%$ \\
\hline & Nein & $9,80 \%$ & $27,60 \%$ & $33,80 \%$ & $13,30 \%$ & $36,00 \%$ & $9,10 \%$ & $21,60 \%$ \\
\hline \multirow{2}{*}{$\begin{array}{l}\text { Vorgehen, } \\
\text { regungslose Person }\end{array}$} & Richtig & $85,60 \%$ & $90,00 \%$ & $73,20 \%$ & $87,10 \%$ & $92,60 \%$ & $90,90 \%$ & $86,60 \%$ \\
\hline & Falsch & $13,80 \%$ & $9,80 \%$ & $26,30 \%$ & $12,60 \%$ & $7,10 \%$ & $9,10 \%$ & $13,10 \%$ \\
\hline \multirow[t]{2}{*}{ Notrufnummer } & Richtig & $98,40 \%$ & $96,50 \%$ & $97,70 \%$ & $95,90 \%$ & $99,40 \%$ & $100,00 \%$ & $98,00 \%$ \\
\hline & Falsch & $1,30 \%$ & $3,50 \%$ & $2,30 \%$ & $4,10 \%$ & $0,60 \%$ & $0,00 \%$ & $2,00 \%$ \\
\hline \multirow{2}{*}{$\begin{array}{l}\text { Wann Beginn der } \\
\text { Herzdruckmassage }\end{array}$} & Richtig & $84,30 \%$ & $87,70 \%$ & $82,60 \%$ & $85,70 \%$ & $89,70 \%$ & $83,60 \%$ & $85,60 \%$ \\
\hline & Falsch & $15,40 \%$ & $12,10 \%$ & $16,90 \%$ & $12,20 \%$ & $10,30 \%$ & $12,70 \%$ & $13,30 \%$ \\
\hline \multirow{2}{*}{$\begin{array}{l}\text { Überprüfung der } \\
\text { Atmung }\end{array}$} & Richtig & $84,30 \%$ & $88,10 \%$ & $71,80 \%$ & $86,70 \%$ & $88,70 \%$ & $92,70 \%$ & $85,40 \%$ \\
\hline & Falsch & $15,40 \%$ & $11,70 \%$ & $27,20 \%$ & $13,30 \%$ & $10,90 \%$ & $5,50 \%$ & $14,00 \%$ \\
\hline \multirow[t]{2}{*}{ Funktion, Herz } & Richtig & $98,70 \%$ & $99,40 \%$ & $83,10 \%$ & $98,30 \%$ & $98,10 \%$ & $98,20 \%$ & $96,00 \%$ \\
\hline & Falsch & $1,00 \%$ & $0,60 \%$ & $15,00 \%$ & $1,70 \%$ & $1,90 \%$ & $0,00 \%$ & $3,40 \%$ \\
\hline \multirow{2}{*}{$\begin{array}{l}\text { Was ist } \\
\text { Herzdruckmassage }\end{array}$} & Richtig & $86,20 \%$ & $86,60 \%$ & $68,50 \%$ & $90,80 \%$ & $88,70 \%$ & $90,90 \%$ & $85,30 \%$ \\
\hline & Falsch & $13,40 \%$ & $13,40 \%$ & $28,60 \%$ & $8,80 \%$ & $10,00 \%$ & $9,10 \%$ & $13,90 \%$ \\
\hline \multirow[t]{2}{*}{ Handposition } & Richtig & $92,50 \%$ & $85,80 \%$ & $89,70 \%$ & $87,80 \%$ & $97,70 \%$ & $85,50 \%$ & $89,80 \%$ \\
\hline & Falsch & $7,20 \%$ & $14,20 \%$ & $8,90 \%$ & $11,90 \%$ & $1,90 \%$ & $12,70 \%$ & $9,50 \%$ \\
\hline \multirow[t]{2}{*}{ Druckfrequenz } & Richtig & $81,30 \%$ & $52,60 \%$ & $88,30 \%$ & $66,70 \%$ & $92,60 \%$ & $81,80 \%$ & $77,20 \%$ \\
\hline & Falsch & $18,40 \%$ & $47,40 \%$ & $10,30 \%$ & $32,70 \%$ & $7,10 \%$ & $18,20 \%$ & $22,30 \%$ \\
\hline \multirow{2}{*}{$\begin{array}{l}\text { Dauer, Überleben der } \\
\text { Hirnzellen }\end{array}$} & Richtig & $87,50 \%$ & $77,20 \%$ & $92,50 \%$ & $86,40 \%$ & $94,50 \%$ & $87,30 \%$ & $87,60 \%$ \\
\hline & Falsch & $11,50 \%$ & $22,30 \%$ & $5,60 \%$ & $13,60 \%$ & $4,80 \%$ & $12,70 \%$ & $11,80 \%$ \\
\hline
\end{tabular}

und der Durchführung von Wiederbelebungsmaßnahmen richtig beantwortet haben. In der Vergangenheit nahmen bereits $1140(71,2 \%)$ Schüler an einem Wiederbelebungstraining teil. Diese beantworteten die Fragen signifikant häufiger richtig ( $88,8 \%$ vs. $84,1 \% ; p=0,02)$. Insgesamt fühlen sich die Schüler sicher, einen Kreislaufstillstand zu erkennen und Wiederbelebungsmaßnahmen durchzuführen. Unsicherheiten gibt es v. a. im Regierungsbezirk Detmold beim Erkennen einer normalen Atmung. Im Regierungsbezirk Münster fühlen sich mehr als die Hälfte der Schüler unsicher bei der Durchführung der Herzdruckmassage (• Abb. 1).

\section{Dezernentenbefragung}

Die Befragung der Schuldezernenten aus den 5 Regierungsbezirken ergab das Erfordernis, Wiederbelebungsmaßnahmen verpflichtend in den Lehrplan zu integrieren. Um die Schulen bis dahin zur Teilnahme am Projekt zu motivieren, sollte die Wichtigkeit des Themas durch die ärztlichen Partner regelmäBig auf Schulleiterdienstbesprechungen der weiterführenden Schulen dargelegt werden. Die notwendige Schulung von Lehrkräften sollte im Rahmen von kurzen, regelmäßigen Lehrerfortbildungen durchgeführt werden, was z. B. mithilfe von kooperierenden Kliniken in der Nähe erfolgen kann. Die Kopplung mit einem Erste-Hilfe-Kurs ist aufgrund des nur geringen Anteils an Wiederbelebung sowohl zeitlich als auch inhaltlich nicht sinnvoll.

Die im Rahmen des Projekts durchgeführten Konzepte müssen auf einer Informationsplattform zur Verfügung gestellt werden. Hierfür bieten sich z.B. das Bildungsportal NRW (www. schulministerium.nrw.de) und die Seite des Landesprogramms Bildung und Gesundheit an (www.bug-nrw.de) an. Weiterhin muss die Versorgung mit Übungsmaterial hinsichtlich Neubeschaffung und Nachbestückung mit Verbrauchsmaterial für alle interessierten Schulen sichergestellt sein.

\section{Kosten}

Für die Ausstattung aller Schulen mit Wiederbelebungspuppen liegt der Einmalinvestitionsbedarf in NRW zwischen 


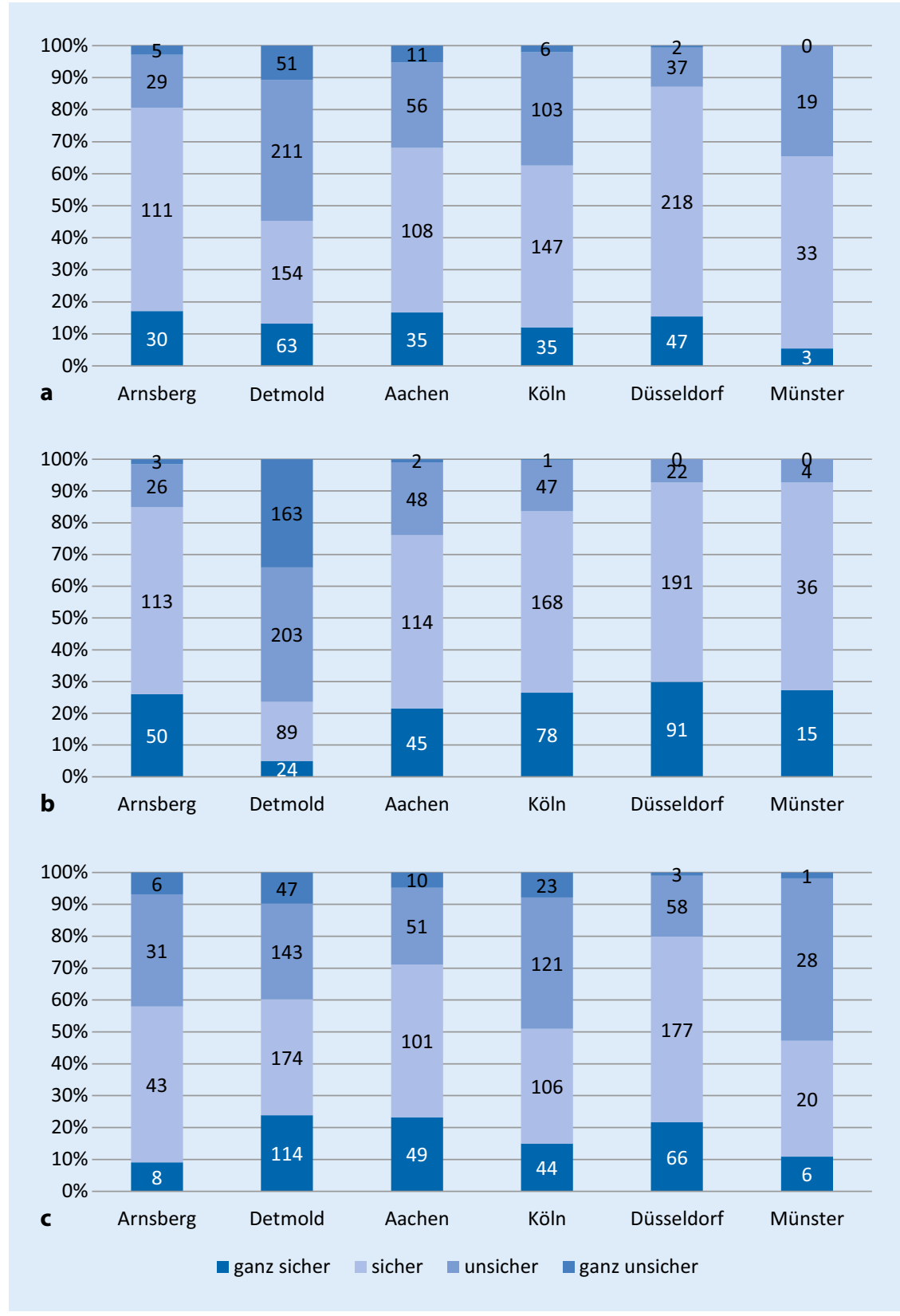

Abb. $1 \Delta$ a Kreislaufstillstand erkennen, b normale Atmung erkennen, c Herzdruckmassage durchführen

4 und 6,5 Mio. $€$. Es ist davon auszugehen, dass die Puppen mindestens 5 Jahre eingesetzt werden können. Zusätzlich benötigt man unabhängig vom durchgeführten Trainingskonzept (Lehrer- oder direktes Schülertraining) etwa $2 € /$ Schüler und Training in den ersten 2 bis 3 Jahren. Dies bedeutet, dass man in NRW bei 170.000 Schülern der 8 . Klasse pro Schuljahr rund $340.000 €$ in jedem Haushaltsjahr einplanen muss (Zusatzmaterial online, s. Hinweisbox am Anfang des Beitrags). gend ausgebaut werden. Um eine flächendeckende Durchführung von Wiederbelebungstrainings in Schulen von NRW $\mathrm{zu}$ erreichen, ist eine gesetzlich geregelte Verpflichtung erforderlich, wie bereits in 5 europäischen Ländern erfolgt, bestenfalls bereits ab der Grundschule [7, 15, 20,23]. Bereits 2 Schulstunden/Schuljahr reichen aus [8], um eine nachhaltige Verbesserung der Kenntnisse in Wiederbelebung zu erreichen. Bis zur gesetzlichen Verpflichtung sollten bereits jetzt vorliegende Konzepte zur Durchführung von Lehrer- und Schülerausbildung genutzt und durch das Ministerium für Schule und Bildung vorangetrieben werden. Auch ist das kontinuierliche Aufzeigen der Wichtigkeit des Themas in Leitungsbesprechungen erforderlich.

Sowohl die Durchführung durch Lehrer als auch durch Ärzte zeigte sich effektiv [8, 19], ein kompaktes Training aller Schüler einer Schule an einem Tag ist nur mit dem Aachener Konzept erfolgt. Hierfür war jedoch auch der größte Material- und Personaleinsatz erforderlich. Zur Durchführung aller anderen Konzepte sind regelmäßige, speziell auf die Wiederbelebung ausgerichtete Lehrerfortbildungen erforderlich, um Motivation und Sicherheit zur Durchführung der Wiederbelebungstrainings $\mathrm{zu}$ erreichen $[11,26]$. Hier haben verschiedene Angebote unterschiedlicher ärztlicher Partner genauso wie vorherige Untersuchungen [23] gezeigt, dass eine erfolgreiche Umsetzung mit verschiedenen regionalen Konzepten möglich ist. Die erfolgreiche und nachhaltige Durchführung von Wiederbelebungstrainings bedarf mindestens eines motivierten Lehrers an einer Schule.

Da Erste-Hilfe-Kurse nicht auf Wiederbelebung fokussieren und hier auch häufig trotz 9-stündiger Dauer Zeit, ausreichend Material und Motivation für das Thema fehlen [33], ist die Kombination von Multiplikatorenschulungen für Wiederbelebungstrainings mit der Ausbildung von Ersthelfern nicht sinnvoll, auch wenn gerade Lehrer auf diesem Gebiet regelmäßige Schulungen benötigen [32]. Bekräftigt wird dies durch die Tatsache, dass in Deutschland jeder, der einen Führerschein besitzt einen Erste-Hilfe-Kurs hat und trotzdem nur 
sehr wenige Menschen mit Laienreanimationsmaßnahmen beginnen. Lehrer können bereits in einer Stunde [8] zu Multiplikatoren ausgebildet werden [11], um dann mit den vorhandenen Konzepten auf Schüler zugeschnittene Trainings von 45-minütiger [14] bis max. 90-minütiger Dauer effektiv und nachhaltig [34] durchzuführen. An dieser Stelle muss den Schulen nochmals kommuniziert werden, dass für die Durchführung eines Wiederbelebungstrainings weder die Teilnahme an einem Erste-Hilfe-Kurs noch der Erwerb eines Ausbilderscheins Erste Hilfe erforderlich ist. Sofern die Hilfsorganisationen über die Bundesarbeitsgemeinschaft (BAGEH) bzw. über die Landesarbeitsgemeinschaften Erste Hilfe (LAGEH) ein speziell auf Wiederbelebung fokussiertes Curriculum wie „Herzensretter“ anbieten [17], wäre die Übernahme von Schulungen für Lehrer oder sogar Schüler denkbar, nicht jedoch mit den üblichen Erste-HilfeKursen. Gleiches gilt für das Programm „Retten macht Schule“ der Björn-SteigerStiftung [5].

Die Entwicklung eines eigenen Konzeptes wäre für die Schulen extrem aufwendig. Viele Materialen sind jedoch bereits erstellt und über Online-Plattformen von DGAI, BDA und GRC für interessierte Schulen jeglicher Schulform jederzeit und ohne Mehraufwand zugänglich. Insbesondere die interdisziplinäre Arbeitsgruppe aus Detmold entwickelt weitere Lehrmaterialen und versucht zudem, Reanimationstrainings in den Routineunterricht zu integrieren. Aus vorgenannten Gründen empfehlen die Autoren den Schulen, ein für sie geeignetes, erprobtes Konzept auszuwählen und dies mithilfe von geschulten Lehrern nachhaltig und kostengünstig zu nutzen.

Als Basis der Laienreanimation kann die alleinige Durchführung der Herzdruckmassage vermittelt werden und sinnvoll sein [21, 28], eine Erweiterung um Beatmung und den automatischen externen Defibrillator ist aufbauend möglich [29]. Die Unsicherheiten der Schüler im Regierungsbezirk Detmold, normale Atmung zu erkennen, lässt sich dadurch erklären, dass die Befragung bei diesen Schülern mehrere Monate nach den Trainings durchgeführt wurde und bis dahin Unsicherheiten wieder zugenommen hatten. Die Autoren halten es daher für sinnvoll, dass langfristig Schüler mehrmals in ihrer Laufbahn ein Reanimationstraining erhalten. Dies wird durch die signifikant besseren Ergebnisse der Schülerbefragung nach bereits in der Vergangenheit erfolgter Teilnahme an einem Training bestärkt. Vorherige Studien zeigen, dass die Kraft zur Durchführung der Herzdruckmassage ab der 7. Klasse ausreicht, die Herangehensweise jedoch bereits deutlich früher geschult werden kann [30].

Voraussetzung für eine erfolgreiche Implementierung von Wiederbelebungstrainings in Schulen ist die Finanzierung des notwendigen Materials. Aufgrund dessen ist eine Fortführung der bereits aus Spendengeldern begonnenen flächendeckenden Beschaffung von Wiederbelebungspuppen, inklusive Zubehör für alle Schulen, erforderlich. Diesbezüglich konnte sich die Beschaffung von Klassensätzen gegenüber einer Schenkung von einer Puppe an jeden Schüler durchsetzen. Ein Klassensatz bietet den Vorteil der Wiederverwendbarkeit, während eine Puppe pro Schüler eine Weitergabe der Kenntnisse im familiären Umfeld der Schüler ermöglicht [18, 25], jedoch puppenabhängig ohne Demonstrationsmöglichkeit der richtigen Drucktiefe. Hierin könnte die Unsicherheit der Schüler des Regierungsbezirks Münster in Bezug auf die Durchführung der Herzdruckmassage begründet sein.

Zusammenfassend können Menschenleben nur dann gerettet werden, wenn Wiederbelebungsmaßnahmen in Schulen in NRW durch das Ministerium für Schule und Bildung gesetzlich vorgeschrieben und koordiniert werden und von den vorhandenen Konzepten mit der notwendigen Finanzierung Gebrauch gemacht wird.

\section{Fazit}

- Eine gesetzliche Verpflichtung und Finanzierung von Wiederbelebungstrainings nach einem Stufenkonzept ist unerlässlich für eine flächendeckende Durchführung.
- Die Schulung von Lehrern sollte gezielt auf Wiederbelebung ausgerichtet sein und kann nicht durch eine traditionelle Erste-Hilfe-Schulung ersetzt werden.

- Es muss nicht jede Schule nach dem gleichen Konzept Wiederbelebungstrainings durchführen, da ausreichend effektive Konzepte zur Verfügung stehen.

- Jede Schule sollte das am besten zu ihr passende Konzept auswählen.

- Die Errichtung einer Informationsplattform ist erforderlich, auf welcher die vorhandenen Konzepte von den Schulen abgerufen werden können.

\section{Korrespondenzadresse}

Dr. med. Marc Felzen

Klinik für Anästhesiologie, Medizinische

Fakultät, RWTH Aachen University, Uniklinik

RWTH Aachen

Pauwelsstr. 30, 52074 Aachen, Deutschland

mfelzen@ukaachen.de

Funding. Open Access funding enabled and organized by Projekt DEAL.

\section{Einhaltung ethischer Richtlinien}

Interessenkonflikt. M. Felzen, H. Schröder, S.K. Beckers, B.W. Böttiger, N. Rott, R. Koch-Schultze, S. Wingen, A. Meißner, I. Santowski, O. Picker, N. Rahe-Meyer, R. Dumcke, C. Wegner, H. van Aken A. Gottschalk, O. Weber und R. Rossaint geben an, dass kein Interessenkonflikt besteht.

Für diesen Beitrag wurden von den Autoren keine Studien an Menschen oder Tieren durchgeführt. Für die aufgeführten Studien gelten die jeweils dort angegebenen ethischen Richtlinien.

Open Access. Dieser Artikel wird unter der Creative Commons Namensnennung 4.0 International Lizenz veröffentlicht, welche die Nutzung, Vervielfältigung, Bearbeitung, Verbreitung und Wiedergabe in jeglichem Medium und Format erlaubt, sofern Sie den/die ursprünglichen Autor(en) und die Quelle ordnungsgemäß nennen, einen Link zur Creative Commons Lizenz beifügen und angeben, ob Änderungen vorgenommen wurden.

Die in diesem Artikel enthaltenen Bilder und sonstiges Drittmaterial unterliegen ebenfalls der genannten Creative Commons Lizenz, sofern sich aus der Abbildungslegende nichts anderes ergibt. Sofern das betreffende Material nicht unter der genannten Creative Commons Lizenz steht und die betreffende Handlung nicht nach gesetzlichen Vorschriften erlaubt ist, ist für die oben aufgeführten Weiterverwendungen des Materials die Einwilligung des jeweiligen Rechteinhabers einzuholen. 
Weitere Details zur Lizenz entnehmen Sie bitte der Lizenzinformation auf http://creativecommons.org/ licenses/by/4.0/deed.de.

\section{Literatur}

1. Anästhesiologie \& Intensivmedizin (2019) Abstract - Deutscher interdisziplinärer Notfallmedizin Kongress. https://www.ai-online.info/supplements/ 2019/05-2019.html.Zugegriffen: 10. Mai 2020

2. BASS (2020) Laienreanimation an Schulen in Nordrhein-Westfalen. https://bass.schul-welt.de/ 16926.htm. Zugegriffen: 14. Mai 2020

3. Bayerisches Staatsministerium für Unterricht und Kultus (2020) Erste-Hilfe-Ausbildung von Schülern wird weiterentwickelt. https:// www.km.bayern.de/lehrer/meldung/6648/ erste-hilfe-ausbildung-von-schuelern-wirdweiterentwickelt.html.Zugegriffen: 14.Sept. 2020

4. BDA, DGAI (2020) Ein Leben Retten. https://www. einlebenretten.de/.Zugegriffen: 14. Sept. 2020

5. Björn Steiger Stiftung (2020) Retten macht Schule. https://www.steiger-stiftung.de/initiativen/ kampf-dem-herztod/retten-macht-schule. Zugegriffen: 10. Mai 2020

6. Bohn A, Lukas RP, Breckwoldt J et al (2015) „Kids save lives": why schoolchildren should train in cardiopulmonary resuscitation. Curr Opin Crit Care 21:220-225. https://doi.org/10.1097/MCC. 0000000000000204

7. Bohn A, Van Aken H, Lukas RP et al (2013) Schoolchildren as lifesavers in Europe-training in cardiopulmonary resuscitation for children. Best Pract Res Clin Anaesthesiol 27:387-396. https:// doi.org/10.1016/j.bpa.2013.07.002

8. Bohn A, Van Aken HK, Möllhoff T et al (2012) Teaching resuscitation in schools: annual tuition by trained teachers is effective starting at age 10. A four-year prospective cohort study. Resuscitation 83:619-625. https://doi.org/10. 1016/j.resuscitation.2012.01.020

9. Böttiger BW, Becker LB, Kern KB etal (2020) BIGFIVE strategies for survival following out-of-hospital cardiac arrest. Eur J Anaesthesiol. https://doi.org/ 10.1097/EJA.0000000000001247

10. Böttiger BW, Lockey A (2018) Jeder Mensch überall auf derWelt-kann ein Leben retten. Notfall Rettungsmed. https://doi.org/10.1007/s10049018-0498-4

11. Böttiger BW, Lockey A, Georgiou M et al (2020) KIDS SAVE LIVES: ERC position statement on schoolteachers' education and qualification in resuscitation. Resuscitation 151:87-90. https:// doi.org/10.1016/j.resuscitation.2020.04.021

12. Böttiger BW, Semeraro F, Altemeyer K-H et al (2017) KIDS SAVE LIVES: school children education in resuscitation for Europe and the world. Eur J Anaesthesiol 34:792-796. https://doi.org/10. 1097/EJA.0000000000000713

13. Dirks B, Wingen S, Rücker G et al (2019) Modularer Lehrerausbildungskurs des Deutschen Rates für Wiederbelebung (GRC) für den Wiederbelebungsunterricht in Schulen. Notfall Rettungsmed 22:334-338. https://doi.org/10.1007/s10049 019-0609-x

14. Felzen M, Lambrecht L, Beckers SK et al (2018) Konzept und Evaluation eines 45-minütigen BLS-Trainings an Schulen. Notfall Rettungsmed 21:367-373. https://doi.org/10.1007/s10049017-0404-5

15. Fleischhackl R, Nuernberger A, Sterz F et al (2009) School children sufficiently apply life supporting first aid: a prospective investigation. Crit Care 13:R127.https://doi.org/10.1186/cc7984

16. Gräsner J-T, Wnent J, Herlitz J et al (2020) Survival after out-of-hospital cardiac arrest in Europe-results of the Eureca TWO study. Resuscitation 148:218-226. https://doi.org/10. 1016/j.resuscitation.2019.12.042

17. Initiative \#HERZENSRETTER (2020) Herzensretter. https://www.herzensretter.info. Zugegriffen: 10. Mai 2020

18. Isbye DL, Rasmussen LS, Ringsted C, Lippert FK (2007) Disseminating cardiopulmonary resuscitation training by distributing 35,000 personal manikins among school children. Circulation 116:1380-1385. https://doi.org/10.1161/ CIRCULATIONAHA.107.710616

19. Jiménez-Fábrega $X$, Escalada-Roig X, Sánchez M et al (2009) Results achieved by emergency physicians in teaching basic cardiopulmonary resuscitation to secondary school students. Eur J Emerg Med 16:139-144. https://doi.org/10.1097/ MEJ.0b013e32831f47b3

20. Jones I, Whitfield R, Colquhoun $M$ et al (2007) At what age can schoolchildren provide effective chest compressions? An observational study from the Heartstart UK schools training programme. BMJ 334:1201. https://doi.org/10.1136/ bmj.39167.459028.DE

21. Kanstad BK, Nilsen SA, Fredriksen K (2011) CPR knowledge and attitude to performing bystander CPR among secondary school students in Norway. Resuscitation 82:1053-1059. https://doi.org/10. 1016/j.resuscitation.2011.03.033

22. Klinik und Poliklinik für Anästhesiologie und Intensivtherapie - Rostocker Simulationsanlage und Notfallausbildungszentrum (2019) Retten macht Schule. https://rosana.med.uni-rostock.de/ ueber-uns/retten-macht-schule/.Zugegriffen: 21. März 2019

23. Lukas R-P, Bohn A, Möllhoff T, Van Aken HK (2013) Teaching resuscitation in schools: „the earlier, the better..... Anasthesiol Intensivmed Notfallmed Schmerzther 48:552-556. https://doi. org/10.1055/s-0033-1355236

24. Malta Hansen C, Zinckernagel L, Ersbøll AK et al (2017) Cardiopulmonary resuscitation training in schools following 8 years of mandating legislation in Denmark: a nationwide survey. J Am Heart Assoc. https://doi.org/10.1161/JAHA.116.004128

25. Møller Nielsen A, Lou Isbye D, Knudsen Lippert $F$ Rasmussen LS (2012) Engaging a whole community in resuscitation. Resuscitation 83:1067-1071. https://doi.org/10.1016/j.resuscitation.2012.04. 012

26. Mpotos N, Vekeman E, Monsieurs $\mathrm{K}$ et al (2013) Knowledge and willingness to teach cardiopulmonary resuscitation: a survey amongst 4273 teachers. Resuscitation 84:496-500. https:// doi.org/10.1016/j.resuscitation.2013.01.023

27. Plant N, Taylor K (2013) How best to teach CPR to schoolchildren: a systematic review. Resuscitation 84:415-421. https://doi.org/10. 1016/j.resuscitation.2012.12.008

28. Rea TD, Fahrenbruch C, Culley L et al (2010) CPR with chest compression alone or with rescue breathing. N Engl J Med 363:423-433. https://doi. org/10.1056/NEJMoa0908993

29. RückerG, DirksB, WingenS, BöttigerBW (2019) Kids Save Lives-Modularer Lehrerausbildungskurs des Deutschen Ratesfür Wiederbelebung (GRC) für den Schulunterricht in Wiederbelebung. Notfall Rettungsmed 22:181-181. https://doi.org/10. 1007/s10049-019-0598-9
30. Rücker G, Schubert J, Scheeren T, NöldgeSchomburg G (2010) Wiederbelebungsunterricht bei Schülern: Ab der siebten Klasse sinnvoll. Dtsch Arztebl 107(11):A-492-C-422

31. Schulsanitätsdienst (2020) LRL-Kurse. https:// www.schulsanitaetsdienst.com/termine/Irlkurse/.Zugegriffen: 12 . Mai 2020

32. Spiegel Online (2019) Entscheidung des Bundesgerichtshofs: Sportlehrer sind zu Erster Hilfe verpflichtet

33. Wagner $P$, Lingemann C, Arntz H-R, Breckwoldt J (2014) Official lay basic life support courses in Germany: is delivered content up to date with the guidelines? An observational study. Emerg Med J. https://doi.org/10.1136/emermed-2014-203736

34. Wingen S, Schroeder DC, Ecker $\mathrm{H}$ et al (2018) Self-confidence and level of knowledge after cardiopulmonary resuscitation training in 14 to 18-year-old schoolchildren: a randomisedinterventional controlled prospective study in secondary schools in Germany. Eur J Anaesthesiol. https://doi.org/10.1097/EJA.0000000000000766

35. Wnent J, Jantzen T, Fischer M et al (2018) Jahresbericht des Deutschen Reanimationsregisters Außerklinische Reanimation 
Hier steht eine Anzeige.

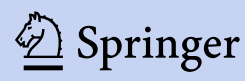

